\title{
Symmetric Plantar Melanocytic Nevi - Site-Specific Phenomenon or Pure Chance
}

\section{Sven Krengel ${ }^{*}$ and $\mathrm{O}$ Haase}

Dermatological Group Practice, Lübeck, Germany

*Corresponding author: Sven Krengel, Dermatological Group Practice, Lübeck, Germany, Tel: 0049451 81551; E-mail: sven.krengel@uk-sh.de Received date: May 24, 2017; Accepted date: June 22, 2017; Published date: June 30, 2017

Copyright: @ 2017 Krengel S, et al. This is an open-access article distributed under the terms of the Creative Commons Attribution License, which permits unrestricted use, distribution, and reproduction in any medium, provided the original author and source are credited.

\begin{abstract}
Background: Predilection sites of melanocytic nevi are highly variable; however, individual patterns (e.g. preponderant involvement of extremities, trunk, etc.) are often noticed. A symmetric appearance of nevi on both sides of the body has not been reported.

Objective: Guided by an initial, incidental observation of symmetrically arranged plantar nevi, a prospective survey of palmoplantar nevi was carried out.

Methods: Between January 2016, and January 2017, 886 patients underwent a full-body examination for skin cancer screening. Special attention was paid to the pigmentary characteristics of the palms and soles.

Results: $12 / 886$ patients exhibited symmetrically or nearly symmetrically distributed plantar nevi (1.35\%; mean age 45 years; male-female ratio 1:1). The nevi were mostly situated on the medial part of the plantar arch (9/12 cases; $75 \%$ ). Remarkably, not a single case of symmetric nevi was identified on the palms.

Conclusion: Arguments in favour of the specifity of the finding are: 1) the lack of comparable findings in palmar skin, i.e., in a similar histological compartment and 2) the predominant localization in the foot arch. Symmetric plantar nevi appear to be a rare, but not a random event. Possible underlying causes of this phenomenon are discussed.
\end{abstract}

Keywords: Melanocytic nevi; Acral palmoplantar symmetry

\section{Introduction}

Acral melanocytic nevi arise on the palms and soles. Nevi on these body sites differ significantly from other types of nevi with respect to epidemiology, clinico-dermoscopic appearance and by their relationship to acral lentiginous melanoma.

By contrast to common melanocytic nevi in other locations, there is no clear racial predilection [1]. Acral nevi are present in 3-9\% of the Caucasian population and are more commonly found in the second and third decades [2,3]. Incidences from some studies were higher in dark-skinned races [4] however, without histological confirmation acral nevi may be confounded with volar melanotic macules. Eruptive nevi with a predilection for the palms and soles may arise during immunosuppressive treatment in patients with cancer, autoimmune disorders, and in transplant recipients $[5,6]$.

Palmoplantar nevi clinically present as irregular brown macules that follow the dermatoglyphic pattern of volar skin. Dermoscopy greatly facilitates the differentiation of acral nevi from acral melanoma: in benign lesions, pigment is predominantly located to the dermal glyphic furrows, whereas it is often accentuated along the dermatoglyphic ridges in melanoma [7].

Histopathologically, besides the typical features of volar skin, acral nevi often show an asymmetric silhouette and intraepidermal upward spread of melanocytes, making these lesions facultative melanoma simulators. Even if acral nevi share epidemiological findings with acral-lentiginous melanoma, there is yet no indication that any single acral nevus has a higher risk to turn malignant than nevi at other locations. However, regarding their role as risk indicators [8] and in view of the serious prognosis of acral melanoma, regular monitoring of acral nevi is necessary. Excision is recommended if atypical clinical features raise the suspicion of melanoma.

In 2015, the authors made the incidental observation of symmetrically distributed plantar nevi. Since then, similar cases were systematically collected.

\section{Methods}

In a dermatological group practice in Northern Germany, patients presenting for skin cancer examinations were prospectively screened for 12 months. This preventive screening took place within the German skin cancer early detection programme, which has been legally introduced in 2008. 886 patients between 4 and 91 years were examined by two experienced dermatologists $(\mathrm{OH}, \mathrm{SK})$ for suspicious nevi and skin cancer between January, 2016 and January, 2017. Based on his earlier observations, the author paid special attention to acral nevi and documented every case of bilateral occurrence of palmar or plantar nevi with a symmetric or near-symmetric distribution. The nevi were designated symmetric or near-symmetric when the location differed $\leq 3 \mathrm{~cm}$, compared to the other side. 
Page 2 of 3

\section{Results}

In 886 patients that underwent a full-body examination, the author identified 12 patients with symmetrically or nearly symmetrically distributed plantar nevi (1.35\%; mean age 45 years; male-female ratio 1:1) (Figures 1a-1g).

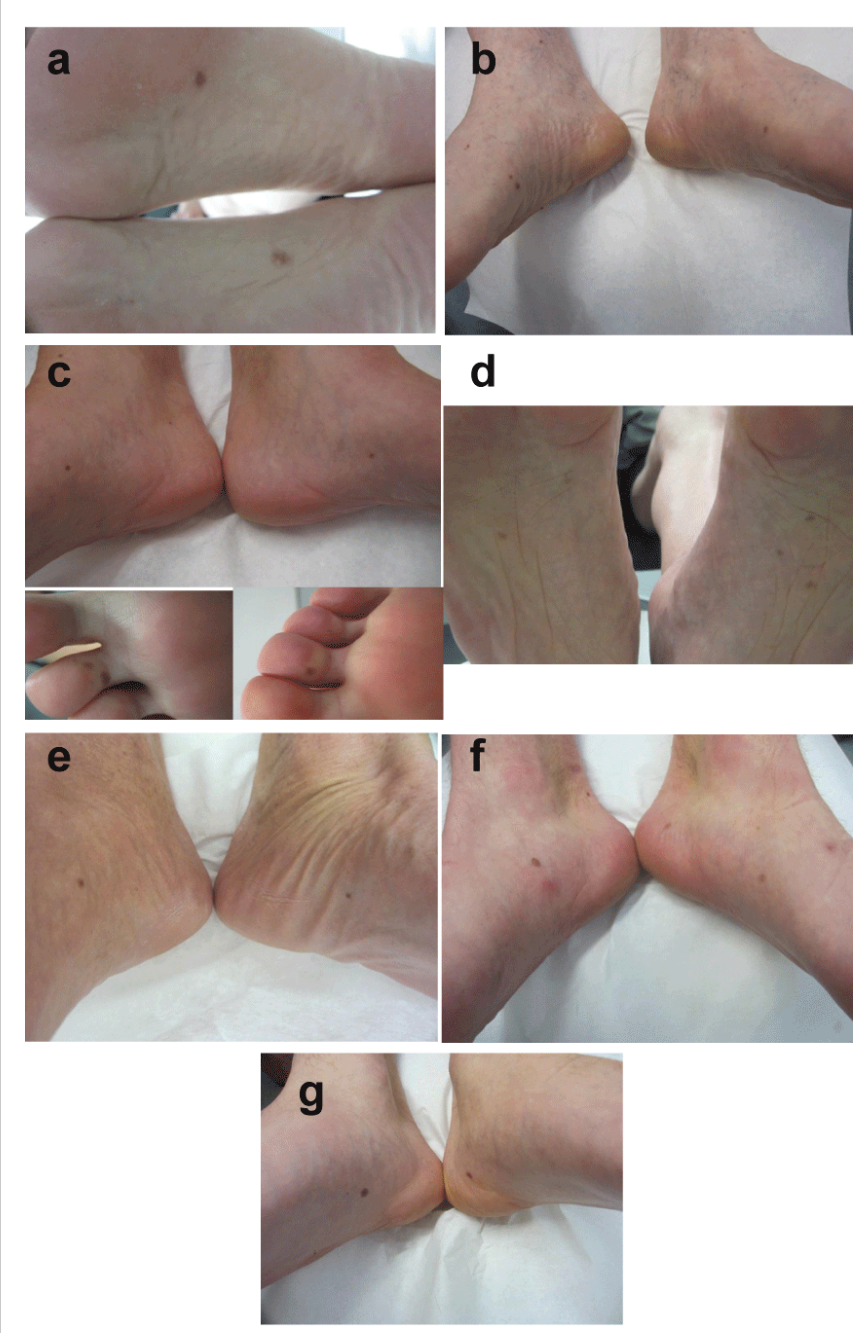

Figure 1: Symmetric plantar nevi. a) 48 year old male patient, b) 62 year old male patient, c) 48 year old female patient, d) 76 year old male patient, e) 70 year old male patient, f) 38 year old male patient, g) 16 year old male patient.

The nevi were mostly situated on the medial part of the plantar arch (9/12 cases; 75\%). In two patients, a symmetric arrangement of nevi was noticed on the plantar aspect of the toes. Remarkably, not a single case of symmetric palmar nevi was identified. Except for one nevus on the 5th toe, no patient reported a congenital nature of the plantar nevus. The plantar nevi had typical clinical and dermoscopical features of acral nevi. The patients were of skin type $1(n=2), 2(n=8), 3(n=1)$, and $4(\mathrm{n}=1)$, according to Fitzpatrick. One of the patients had a congenital reticular hyperpigmentation on the upper thorax. The medical history of the patients showed malignant melanoma $(n=1)$, dysplastic nevi $(n=1)$ and basal cell carcinoma $(n=2)$. One patient was on medication with azathioprine due to neurosarcoidosis $(n=1)$. Two patients reported regular use of sunbeds. One patient had palmoplantar psoriasis.

\section{Discussion}

Palmoplantar ("acral") melanocytic nevi are recognized as a distinct entity. They represent a benign melanocytic proliferation in ridged skin. This type of skin is characterized by its huge corneal layer, the lack of hair follicles and abundancy of eccrine sweat glands. Certain types of keratin (K6, K9, K16, K17) are specifically expressed in palmoplantar skin [9]. An influence of the epidermal or adnexal microenvironment on nevogenesis is possible, but not well-established [10]. The rare entity of epidermolysis-bullosa-nevi might represent a link between an epithelial skin disease and nevogenesis; however, this eruption is more likely related to components of the blister fluid or to secondary inflammatory changes [11].

Palmoplantar nevi, unlike other subtypes of melanocytic nevi, have not yet been assigned a special molecular signature [12]. For acral melanoma, a predisposition to cKIT mutations [13] and a relative paucity of BRAF mutations [14] has been shown.

Acral melanocytic nevi have been grouped together with nevi of the embryonic milk line (breast, axillae, umbilicus, genitalia), flexural areas, ear, and scalp, as facultative histopathological simulators of melanoma [15]. A subtype of melanocytic nevi of the distal lower extremity (ankle region) with architectural disorder has been described [16]. As the symmetric plantar nevi from our series did not show worrisome features, excision was not performed. Therefore, we are unable to comment on histopathological features.

In this study, plantar melanocytic nevi were designated symmetric or near-symmetric in 12 patients. The threshold of $\leq 3 \mathrm{~cm}$ side difference reflects the common perception of symmetry given the average size of adult palms and soles. However, regarding the possible biological significance of the findings, it must be beared in mind that this threshold was arbitrarily chosen.

The finding of a symmetric or near-symmetric distribution of plantar melanocytic nevi has, to the best of our knowledge, not yet been reported. However, at least one earlier report of plantar nevi shows a picture with a remarkable degree of symmetry [6]. Symmetric plantar nevi appear to be a rare, but not a random event. This is evident regarding the lack of any similar finding in palmar skin, i.e., in a comparable histological compartment with a presumably similar pathogenesis. The predominant localization in the foot arch underscores the specificity of the finding. This predilection site also speaks against physical pressure as an exogenous trigger of symmetric plantar nevi.

Symmetrically arranged skin conditions are a frequent observation in inflammatory skin diseases. E.g. symmetrically distributed psoriatic plaques on either elbows or knees are a very common finding. This fact is mostly related to the predilection of a given entity to distinct sites of the body. Predilection sites may be based on local anatomical or functional characteristics, on exogenous factors (e.g. sunlight exposure) or on a combination of endogenous and exogenous causes.

It has been proposed that predilection sites might represent a consequence of a ubiquitous somatic mosaicism [17]. In the case of neoplastic (benign or malignant) skin conditions, a symmetric distribution of two or more lesions is much less frequent. One case of strikingly symmetric facial epidermal nevi has been reported [18]. The authors attributed this finding to the origin of the condition from the 
lines of Blaschko. Regardless of the rarity of reports, it is well-known that neoplastic skin conditions do have predilection sites. Males and females differ regarding preferentially affected body sites, for benign melanocytic nevi [3] as well as for melanoma [19].

In an earlier study, the author reported on predilection sites of subtypes of melanocytic nevi (flat nevi, Miescher nevi, Unna nevi) [20]. Malignant melanoma - by contrast to most epithelial skin cancers - often develops at sites that are not explained by the mere quantity of cumulative ultraviolet radiation doses [14]. All these facts suggest that the localization of a given melanocytic proliferation importantly depends on endogenous local factors.

Moreover, patients frequently report that "birthmarks" (e.g. melanocytic nevi, café- au-lait-macules, vascular nevi) manifest at the same body site in members of consecutive generations of one family. A scientific explanation for these phenomena has not been found and it is a constant matter of debate whether such cases represent pure chance. As early as 1924, Siemens demonstrated by meticulous examinations of 136 twin pairs of school age that the localization of melanocytic and vascular nevi is not genetically determined [21].

\section{Conclusion}

To conclude, symmetric plantar nevi represent a peculiar, yet unexplained phenomenon. Hopefully, our present report will prompt future investigators to pay attention to this phenomenon. Genotypeto-phenotype correlations might enable a better understanding of cutaneous predilection sites in the future.

\section{References}

1. Cullen SI (1962) Incidence of nevi. Report of survey of the palms, soles and genitalia of 10,000 young men. Arch Dermatol 86: 40-43.

2. Kopf AW (1964) Pigmented nevi of the palms and soles. Med Rec Ann 57: 511-514.

3. MacKie RM, English J, Aitchison TC, Fitzsimons CP (1985) The number and distribution of benign pigmented moles (melanocytic naevi) in a healthy British population. Br J Dermatol 113: 167-174.

4. Coleman WP, Gately LE, Krementz AB, Reed RJ, Krementz ET (1980) Nevi, lentigines and melanomas in blacks. Arch Dermatol 116: 548-551.

5. Hughes BR, Cunliffe WJ, Bailey CC (1989) Excess benign melanocytic naevi after chemotherapy for malignancy in childhood. BMJ 299: 88-91.
6. Woodhouse J, Maytin EV (2005) Eruptive nevi of the palms and soles. J Am Acad Dermatol 52: S96-96S100.

7. Steiner A, Binder M, Schemper M, Wolff K, Pehamberger H (1993) Statistical evaluation of epiluminescence microscopy criteria for melanocytic pigmented skin lesions. J Am Acad Dermatol 29: 581-588.

8. Green A, McCredie M, MacKie R, Giles G, Young P, et al. (1999) A casecontrol study of melanomas of the soles and palms (Australia and Scotland). Cancer Causes Control 10: 21-25.

9. McLean WH (2003) Genetic disorders of palm skin and nail. J Anat 202: 133-141.

10. Krengel S (2005) Nevogenesis--new thoughts regarding a classical problem. Am J Dermatopathol 27: 456-465.

11. Lanschuetzer CM, Emberger M, Hametner R, Klausegger A, Pohla-Gubo G, et al. (2003) Pathogenic mechanisms in epidermolysis bullosa naevi. Acta Derm Venereol 83: 332-337.

12. Ross AL, Sanchez MI, Grichnik JM (2011) Molecular nevogenesis. Dermatol Res Pract 2011: 463184.

13. Curtin JA, Busam K, Pinkel D, Bastian BC (2006) Somatic activation of KIT in distinct subtypes of melanoma. J Clin Oncol 24: 4340-4346.

14. Viros A, Fridlyand J, Bauer J, Lasithiotakis K, Garbe C, et al. (2008) Improving melanoma classification by integrating genetic and morphologic features. PLoS Med 5: e120.

15. Mason AR, Mohr MR, Koch LH, Hood AF (2011) Nevi of special sites. Clin Lab Med 31: 229-242.

16. Khalifeh I, Taraif S, Reed JA, Lazar AF, Diwan AH, et al. (2007) A subgroup of melanocytic nevi on the distal lower extremity (ankle) shares features of acral nevi, dysplastic nevi and melanoma in situ: a potential misdiagnosis of melanoma in situ. Am J Surg Pathol 31: 1130-1136.

17. Cho RJ (2010) Rethinking the prosaicism of mosaicism; is it in us? Exp Dermatol 19: 1037-1039.

18. Shah KN, Honig PJ, Yan AC (2007) Bilateral symmetric facial epidermal nevus. J Am Acad Dermatol 56: S51-53.

19. Wallingford SC, Alston RD, Birch JM, Green AC (2011). Increases in invasive melanoma in England, 1979-2006, by anatomical site. Br J Dermatol 165: 859-864.

20. Witt C, Krengel S (2010) Clinical and epidemiological aspects of subtypes of melanocytic nevi (Flat nevi, Miescher nevi, Unna nevi). Dermatol Online J 16: 1 .

21. Siemens HW (1924) Über die Bedeutung der Erbanlagen für die Entstehung der Muttermäler. Archiv für Dermatologie und Syphilis 147: 1-60. 